\title{
Metodología de Evaluación de los Pararrayos de Carburo de Silicio
}

Arnaldo G. Kanashiro ${ }^{1}$, Milton Zanotti $\mathrm{Jr}^{1}{ }^{1}$, Paulo F. Obase ${ }^{1}$ y Wilson R. Bacega ${ }^{2}$

(1) Universidade de São Paulo, Inst. de Eletrotécnica e Energia, Av. Prof. Luciano Gualberto, 1289, 05508-010,São Paulo, SP-Brasil (e-mail: arnaldo@iee.usp.br).

(2) CTEEP - Companhia de Transmissão de Energia Elétrica Paulista, Rua Casa do Ator, 1.155. 04546-004, São Paulo, SP-Brasil.

Recibido Jun. 01, 2009; Aceptado Ago. 05, 2009; Versión Final recibida Sep.30, 2009

\begin{abstract}
Resumen
Este trabajo presenta los resultados de una investigación cuyo objetivo fue el diagnóstico de los pararrayos de carburo de silicio. En el laboratorio fueron realizados ensayos en pararrayos, siendo determinados los valores de tensión disruptiva en frecuencia industrial y de impulso atmosférico. Esos resultados fueron comparados con valores de la corriente de fuga, radiointerferencia y termovisión, obtenidos en mediciones en los mismos pararrayos. Se observó que valores más elevados de la corriente de fuga, en términos de amplitud y componente de tercer armónico, estaban asociados con una mayor degradación en esos pararrayos. Con base en esos resultados, se concluyó que la medición de la corriente de fuga puede proveer informaciones importantes sobre el estado de esos pararrayos. Mediciones del comportamiento de la corriente de fuga a lo largo de los años en las suestaciones pueden ayudar a identificar los pararrayos más degradados.
\end{abstract}

Palabras clave: carburo de silicio, corriente de fuga, pararrayos, subestación

\section{Assessment Methodology of Silicon Carbide Surge Arresters}

\begin{abstract}
This work presents results of a research project dveloped for determining the diagnostic of silicon carbide surge arresters. Tests were performed in these arresters in the laboratory, considering the power frequency spark-over voltage and lightning spark-over voltage tests. The results were compared with values of leakage current, radio-influence voltage test and thermovision, obtained in the same surge arresters. It was observed a reasonable correlation between high values of the leakage current, considering the amplitude and the third harmonic component, and the presence of degradation in the surge arresters. Therefore, it was concluded that leakage current measurements can provide important information about the stage of degradation of the surge arresters. Measurements in the substations during several years could provide the identification, in case of leakage current variations, of deteriorated silicon carbide surge arresters.
\end{abstract}

Keywords: silicon carbide, leakage current, surge arresters, substation 


\section{INTRODUCCIÓN}

En Brasil, hay básicamente dos tipos de pararrayos instalados en el sistema eléctrico de las concesionarias: los pararrayos de carburo de silicio ( $\mathrm{SiC}$ ) y los de óxido de zinc (ZnO). Los primeros son más antiguos y de tecnología obsoleta; el segundo tipo es la evolución del primero y presenta mejor desempeño (Christodoulou et al., 2008; Miyakawa et al., 2008). Los pararrayos de SiC están siendo gradualmente sustituidos por los de $\mathrm{ZnO}$, pues la sustitución inmediata de todos los pararrayos resulta en costos inaceptables para las concesionarias. En un relevamiento hecho en 2004, de los cerca de 19.000 pararrayos instalados en el sistema eléctrico brasileño, de las clases de tensión de $34,5 \mathrm{kV}$ a $750 \mathrm{kV}, 64 \%$ eran de carburo de silicio, con tiempo de instalación promedio de 21 años (Carneiro, 2007). El tiempo de instalación de estos equipos es muy largo, pudiendo presentar anomalías que pueden llevar a una falla. En este caso, es necesario el conocimiento del estado actual de esos equipos, de modo a auxiliar las concesionarias en la elaboración de un programa de sustitución de los pararrayos de $\mathrm{SiC}$, priorizándose la retirada de aquellos que estuvieran más degradados.

Los métodos de diagnóstico de los pararrayos de ZnO (Heinrich y Hinrichen, 2001; Almeida et al., 2009) fueron desarrollados a lo largo de los últimos años; pero, en cuanto a los pararrayos de SiC; no hay un consenso sobre el asunto, tanto que la IEC 60099-5 (2000) no propicia ninguna recomendación al respecto del diagnóstico de los pararrayos de SiC. En ese documento son recomendados diversos métodos para la evaluación de los pararrayos de $\mathrm{ZnO}$; de entre estos, el método que se destaca es el de la medición de la corriente de fuga con análisis de su componente del tercer armónico $\left(3^{\circ} \mathrm{A}\right)$. Este método es particularmente útil porque la corriente de fuga contiene informaciones al respecto de sus componentes internos, no siendo necesaria la retirada de los pararrayos de las instalaciones para ejecución de las mediciones. Esto se vuelve interesante para las concesionarias de energía, pues no hay la necesidad de interrupciones programadas, disminuyendo así los costos de estas evaluaciones. Se resalta que la concesionaria tiene dificultades en la programación de interrupciones en función de recomendaciones muy rígidas de la agencia que reglamenta el sector eléctrico brasileño (Piantini y Janiszewski, 2004). Por tanto, técnicas de diagnóstico de pararrayos que no necesiten de interrupciones son extremadamente deseables.

En la literatura es posible verificar que una parte razonable de las fallas en pararrayos, tanto de $\mathrm{ZnO}$ cuanto de SiC, son atribuidas a la entrada de humedad en los pararrayos (Kannus y Lahti, 2005). Inicialmente, las investigaciones en los pararrayos de $\mathrm{SiC}$ se limitaban a identificar pararrayos con problemas, tomándose por base la comparación de valores obtenidos en ensayos normalizados. Ésto trae como desventaja la necesidad de desenergizar el equipo y llevarlo al laboratorio. Con el desarrollo tecnológico, fue posible adquirir señales de estos equipos sin la necesidad de desenergizarlos en la subestación. La experiencia en la medición de la corriente de fuga en los pararrayos de $\mathrm{ZnO}$ y el desarrollo de la imagen térmica por infrarrojo, conocida como termovisión, contribuyeron para ayudar en el desarrollo de procedimientos para la evaluación de los pararrayos de $\mathrm{SiC}$.

El análisis del componente $3^{\circ} \mathrm{A}$ es una técnica utilizada en los pararrayos de $\mathrm{ZnO}$, y que podría ser aplicada a los pararrayos de SiC, ya que poseen centelladores, activos y parcialmente activos, conectados con los resistores no lineales, por donde pasa la corriente de fuga. El componente $3^{\circ} \mathrm{A}$ puede ser relacionado con la componente resistiva de la corriente de fuga total. El aumento de la componente resistiva causa el aumento de la disipación de la energía en los resistores, lo que puede llevar a la degradación del pararrayos y a su falla, de ahí su importancia para el monitoreo de estos equipos.

Este trabajo presenta los resultados de una investigación realizada en pararrayos de SiC, teniéndose por objetivo el diagnóstico del estado de esos pararrayos. Fueron realizados ensayos en 35 pararrayos, cuatro fabricantes, que fueron seleccionados de un total de 200 puestos a disposición por la concesionaria. Esos pararrayos estuvieron en servicio por varios años. En el trabajo son presentados los resultados de 12 pararrayos, de dos fabricantes, clases de tensión $88 \mathrm{kV}$ y $138 \mathrm{kV}$, que representa el comportamiento de los 35 pararrayos investigados. Los resultados mostraron que valores más elevados de la corriente de fuga, en términos de amplitud y componente de tercer 
armónico, indicaron la existencia de mayor degradación en los pararrayos. A pesar del número reducido de pararrayos ensayados, el material utilizado en los resistores de todos los pararrayos antiguos es el mismo (carburo de silicio), siendo esperado un comportamiento similar en los pararrayos instalados en las subestaciones. De cualquier modo, se trata de un resultado importante que debe ser posteriormente verificado a través de mediciones en mayor número de pararrayos en las concesionarias.

\section{METODOLOGÍA}

Los pararrayos de $\mathrm{ZnO}$ y $\mathrm{SiC}$ poseen elementos no lineales sometidos a la tensión sinusoidal y entonces la corriente de fuga total presenta distorsión. A través de la descomposición de la señal, la corriente de fuga total puede ser expresada como la suma de varias sinusoidales de frecuencias con múltiples enteros de la frecuencia fundamental de la forma de onda original. En Brasil, la frecuencia de la rede es $60 \mathrm{~Hz}$. Las sinusoidales puras son llamadas de armónicos o serie de Fourier. Cuando la degradación de los pararrayos de $\mathrm{ZnO}$ aumenta, hay el aumento de la corriente resistiva y del componente $3^{\circ} \mathrm{A}$ de la corriente de fuga total. La amplitud del componente $3^{\circ} \mathrm{A}$ de la corriente de fuga total varía de acuerdo con el coeficiente de no linealidad de los resistores de ZnO así, es posible monitorear la componente resistiva de la corriente de fuga total a través de la medición del componente $3^{\text {ㅇ }} \mathrm{A}$ (Schei, 2000). El componente $3^{\circ} \mathrm{A}$ es un buen indicativo de alteración de la condición de los resistores de $\mathrm{ZnO}$. En este trabajo, esa medición es aplicada a los pararrayos de $\mathrm{SiC}$.

Considerándose que los pararrayos de $\mathrm{SiC}$ están siendo sustituidos por los de $\mathrm{ZnO}$, es necesario que aquellos más degradados sean priorizados y retirados del servicio antes de su falla. Dentro de ese contexto, fueron investigadas técnicas de diagnóstico utilizándose pararrayos de SiC, los cuales estuvieron en servicio por varios años. Esos pararrayos, clases de tensión $88 \mathrm{kV}$ y $138 \mathrm{kV}$, fueron retirados del campo en función de fallas, reformas en la subestación o como parte de un procedimiento de sustitución gradual de esos equipos. Los pararrayos de SiC de $88 \mathrm{kV}$, fabricante $\mathrm{A}$, y de $138 \mathrm{kV}$, fabricante $\mathrm{B}$, se encontraban en el depósito de la concesionaria de energía eléctrica y fueron transportados al Laboratorio de Alta Tensión. De modo general, eran visibles señales de corrosión en las partes metálicas y, en algunos pararrayos, era posible constatar marcas de descargas, indicando ocurrencia de fallas en el campo. No había un histórico de mediciones que permitiera indicar el envejecimiento de esos pararrayos a lo largo del tiempo. Por tanto, las únicas referencias que podrían ser utilizadas eran los valores de normas y/o catálogos. Esos datos, sin embargo, no siempre estaban disponibles.

Inicialmente, los pararrayos fueron analizados visualmente, procurándose identificar aquellos con evidencias de entrada de humedad y con marcas externas de descargas. Luego de esta fase, el comportamiento de los pararrayos fue analizado considerándose los siguientes ensayos:

Tensión disruptiva de impulso atmosférico con forma de onda normalizada;

Tensión disruptiva a la frecuencia industrial a seco;

Medición de la corriente de fuga;

Medición de radiointerferencia (RIV);

Medición de temperatura (termovisión).

La medición de la corriente de fuga fue realizada en la tensión de operación de los pararrayos (51 kV, fabricante A, $80 \mathrm{kV}$, fabricante B). Esos valores son correspondientes a las tensiones fasetierra del sistema trifásico de $88 \mathrm{kV}$ y de $138 \mathrm{kV}$, respectivamente. Fue utilizado un transformador de alta tensión en el laboratorio. Fueron hechos registros de la corriente de fuga a través de la caída de tensión sobre un resistor colocado entre la base del pararrayos y la tierra del laboratorio. Con un osciloscopio, fueron obtenidas las formas de onda de la tensión aplicada y de la corriente de fuga del pararrayos. La medición de RIV y la termovisión tuvieron por objetivo ayudar en el análisis e interpretación de los resultados de la corriente de fuga y, por tanto, no fueron realizadas en todos los pararrayos. La tensión de radiointerferencia fue medida con el pararrayos energizado en la tensión de operación. La frecuencia y la impedancia de medición fueron iguales a $500 \mathrm{kHz}$ y 300 ohms, respectivamente, que son valores recomendados por la IEC 60099-1 (1999). Una vez finalizada la 
medición de RIV, fueron seleccionados algunos pararrayos para la realización de la termovisión. Las mediciones de temperatura fueron realizadas luego de seis horas de energización de los pararrayos en la tensión de operación. Cada uno de los pararrayos fue dividido en 4 facetas en la dirección longitudinal (del tope hasta la base). En cada faceta fue hecho un termograma en el cual la cámara marca una línea, llamada LI01, donde son registradas las temperaturas a lo largo del pararrayos.

Posteriormente, algunos pararrayos fueron desmontados para verificación de una posible correlación entre los resultados obtenidos en los ensayos y el estado de los elementos internos del equipo. Fue investigada la existencia de marcas de cúmulo de humedad y/o de descargas internas. La medición de la corriente de fuga en pararrayos de SiC resultó en informaciones muy importantes al respecto del estado del pararrayos, principalmente cuando es analizado el componente $3^{\circ} \mathrm{A}$. Ese resultado motivó la realización de medición de la corriente de fuga en pararrayos instalados en la subestación de Paraibuna.

\section{RESULTADOS Y ANÁLISIS}

\section{Características de los pararrayos}

Son mostrados los resultados de las investigaciones realizadas en el laboratorio y en la subestación de Paraibuna. Los pararrayos cedidos por la concesionaria de energía son de dos fabricantes, siendo identificados como fabricante A y fabricante B, cuyas características son mostradas en la Tabla 1. Los pararrayos poseen dos módulos que son montados uno sobre otro. Los módulos de los pararrayos del fabricante A son iguales. Los módulos de los pararrayos del fabricante B son diferentes entre sí.

Tabla 1: Características de los pararrayos.

\begin{tabular}{|c|c|c|c|c|}
\hline Fabricante & $\begin{array}{c}\text { Corriente de } \\
\text { descarga } \\
\text { nominal (kA) }\end{array}$ & $\begin{array}{c}\text { Tensión } \\
\text { Nominal (kV) }\end{array}$ & $\begin{array}{c}\text { Número de } \\
\text { módulos }\end{array}$ & Cantidad \\
\hline A & 10 & 85 & 2 & 6 \\
\hline$B$ & 10 & 132 & 2 & 6 \\
\hline
\end{tabular}

Tensión disruptiva a la frecuencia industrial y de impulso atmosférico

Los ensayos de tensión disruptiva a la frecuencia industrial $(60 \mathrm{~Hz})$ y de tensión disruptiva de impulso atmosférico prueban el comportamiento de los centelladores de los pararrayos frente a las sobretensiones temporarias e impulsivas del sistema, respectivamente. El comportamiento deseable del pararrayos frente a estos ensayos es que el valor de tensión disruptiva de impulso atmosférico sea el menor posible, para garantizar el máximo de protección, y que el valor de tensión disruptiva a la frecuencia industrial sea el mayor posible, para evitar interrupciones indebidas por ocasión de sobretensiones temporales. La Tabla 2 muestra los valores obtenidos de tensión disruptiva de frecuencia industrial y de impulso atmosférico, donde $(R)$ es la indicación de pararrayos reprobados en el ensayo de tensión disruptiva a la frecuencia industrial.

Comparándose los resultados mostrados en la Tabla 2 con aquellos especificados en el catálogo del fabricante y en la norma brasileña NBR 5287 (1988), se puede afirmar que:

i) todos los pararrayos del fabricante A fueron reprobados en el ensayo de tensión disruptiva en frecuencia industrial (A5 y A6 presentaron comportamiento inestable superior a $80 \mathrm{kV}$, no siendo posible la determinación de los valores);

ii) un pararrayos del fabricante B (B5) fue reprobado en el ensayo de tensión disruptiva en frecuencia industrial;

iii) todos los pararrayos fueron aprobados en el ensayo de tensión disruptiva de impulso atmosférico. 
Tabla 2: Valores de tensión disruptiva a la frecuencia industrial y de impulso atmosférico.

\begin{tabular}{|c|c|c|c|}
\hline Pararrayos & Tensión disruptiva & \multicolumn{2}{|c|}{ Tensión disruptiva de impulso atmosférico } \\
\cline { 3 - 4 } (Fab. A y B) & a la frecuencia industrial $(\mathrm{kV})$ & Pol. Pos. (kV) & Pol. Neg. (kV) \\
\hline A1 & $134(\mathrm{R})$ & 182 & 181 \\
\hline A2 & $105(\mathrm{R})$ & 171 & 168 \\
\hline A3 & $85(\mathrm{R})$ & 178 & 178 \\
\hline A4 & $102(\mathrm{R})$ & 172 & 167 \\
\hline A5 & $(\mathrm{R})$ & 173 & 172 \\
\hline A6 & $(\mathrm{R})$ & 173 & 188 \\
\hline B1 & 226 & 382 & 354 \\
\hline B2 & 219 & 374 & 363 \\
\hline B3 & 224 & 364 & 359 \\
\hline B4 & 218 & 340 & 322 \\
\hline B5 & $188(R)$ & 349 & 344 \\
\hline B6 & 233 & 355 & 344 \\
\hline
\end{tabular}

El ensayo de tensión disruptiva a la frecuencia industrial identificó los pararrayos que presentaron desvíos en relación a sus características originales, indicando que hubo degradación en los centelladores de esos pararrayos. Con relación al ensayo de tensión disruptiva de impulso atmosférico, todos los pararrayos fueron aprobados.

\section{Medición de la corriente de fuga}

Los pararrayos sometidos a la medición se encontraban en varios estadios de conservación. Algunos no presentaban cualquier señal externa de degradación mientras otros poseían marcas de descargas. Los valores obtenidos son presentados en la Tabla 3 y fueron organizados, para cada fabricante, en orden creciente del componente $3^{\circ} \mathrm{A}$ de la corriente de fuga total, con excepción de los pararrayos A5 y A6 en los que no fue posible obtenerse el valor de tensión disruptiva a la frecuencia industrial. En la Tabla 3, los parámetros son: $I_{p-p}$ : valor pico a pico de la corriente de fuga; $I_{\text {rms: }}$ : valor eficaz de la corriente de fuga; $I_{p}$ : valor de pico de la corriente de fuga; Ip/Irms: relación entre el valor de pico y el valor eficaz de la corriente de fuga.

Los valores pico a pico $\left(I_{p-p}\right)$ fueron medidos en el osciloscopio. Las formas de onda de la corriente de fuga fueron registradas en planilla electrónica para posterior tratamiento y análisis con el programa computacional PSpice (2008). Con la utilización del programa fue posible obtener los valores eficaces $\left(I_{\mathrm{rms}}\right)$ y la corriente de pico $\left(I_{\mathrm{p}}\right)$. Los valores del componente $3^{\circ}$ A son dados en porcentaje de la componente de frecuencia fundamental $(60 \mathrm{~Hz})$. El campo Desfase $(\Psi)$ se refiere al desfase, en grados, entre la tensión aplicada (frecuencia $60 \mathrm{~Hz}$ ) y la respectiva corriente de fuga total (frecuencia $60 \mathrm{~Hz}$ ) del pararrayos, medida a través del osciloscopio. Este parámetro fue registrado con el objetivo de verificarse una posible relación entre el desfase y el estado de degradación del pararrayos, una vez que valores menores de desfase indican un comportamiento más resistivo de la corriente de fuga total (frecuencia $60 \mathrm{~Hz}$ ).

Los pararrayos $\mathrm{A} 5$ y $\mathrm{A} 6$ presentaron componentes $3^{\circ} \mathrm{A}(5,7 \%$ e $5,0 \%)$ menores que el pararrayos A1 $(6,7 \%)$, pero, las corrientes de fuga total se presentaron con desfases de $39^{\circ}(A 5)$ y $67^{\circ}(A 6)$, en comparación con el desfase de $89^{\circ}$ del pararrayos $A 1$. Este hecho indica que las corrientes de fuga total de los pararrayos A5 y A6 presentaron una característica más resistiva. Con relación a la corriente de fuga total de los pararrayos $A 1, A 2, A 3$ y A4, los valores $I_{p-p}, I_{r m s}$ e $I_{p}$ aumentaron a la medida que la distorsión aumentó. El mismo comportamiento fue obtenido para la relación $\mathrm{I}_{\mathrm{p}} / \mathrm{I}_{\mathrm{rms}}$. El desfase presentó comportamiento inverso, o sea, hubo disminución de esos valores. 
Tabla 3: Resultados de la medición de la corriente de fuga.

\begin{tabular}{|c|c|c|c|c|c|c|c|}
\hline \multirow{2}{*}{ Pararrayos } & \multirow{2}{*}{$\begin{array}{c}\text { Tensión } \\
\text { aplicada } \\
(\mathrm{kV})\end{array}$} & \multicolumn{6}{|c|}{ Corriente de fuga (mA) } \\
\hline & & $I_{p-p}$ & $I_{\text {rms }}$ & $I_{p}$ & $\mathrm{I}_{\mathrm{p}} / \mathrm{I}_{\mathrm{rms}}$ & $\begin{array}{l}3^{\circ} \mathrm{A} \\
(\%)\end{array}$ & $\begin{array}{c}\Psi \\
\text { (arados) }\end{array}$ \\
\hline $\mathrm{A} 1$ & \multirow{6}{*}{51} & 0,325 & 0,131 & 0,172 & 1,450 & 6,7 & 89 \\
\hline $\mathrm{A} 2$ & & 0,445 & 0,147 & 0,213 & 1,449 & 10,1 & 65 \\
\hline A3 & & 0,735 & 0,291 & 0,456 & 1,567 & 24,9 & 54 \\
\hline A4 & & 1,500 & 0,448 & 0,766 & 1,710 & 32,9 & 47 \\
\hline A5 & & 0,669 & 0,234 & 0,357 & 1,526 & 5,7 & 39 \\
\hline A6 & & 0,706 & 0,248 & 0,348 & 1,403 & 5,0 & 67 \\
\hline B1 & \multirow{6}{*}{80} & 0,727 & 0,293 & 0,396 & 1,352 & 5,6 & 73 \\
\hline B2 & & 0,964 & 0,352 & 0,501 & 1,423 & 5,8 & 75 \\
\hline B3 & & 0,691 & 0,250 & 0,346 & 1,382 & 6,8 & 79 \\
\hline B4 & & 0,684 & 0,260 & 0,362 & 1,392 & 6,9 & 68 \\
\hline B5 & & 0,698 & 0,300 & 0,430 & 1,433 & 7,5 & 83 \\
\hline B6 & & 1,620 & 0,628 & 0,823 & 1,311 & 18 & 51 \\
\hline
\end{tabular}

El pararrayos A4 tuvo el mayor valor de corriente, la mayor distorsión armónica (32,9 \%) y el menor desfase $\left(47^{\circ}\right)$, en relación a los valores obtenidos en los pararrayos A1, A2 e A3. Esos resultados sugieren que hay una relación directa entre valores más elevados de la corriente de fuga total y del componente de $3^{\circ}$ A con menores valores de desfase. Esa tendencia puede indicar un mayor estado de degradación del pararrayos.

Con relación al fabricante $\mathrm{B}$, inicialmente son analizados los resultados de los pararrayos B1 a B5. El $\mathrm{B} 5$, el único reprobado en el ensayo de tensión disruptiva a la frecuencia industrial, fue obtenido un valor ligeramente mayor del componente de $3^{\circ}$ A $(7,5 \%)$ en comparación con aquellos registrados en los pararrayos B1, B2, B3 y B4. Por otro lado, el desfase obtenido en el pararrayos B5 fue el mayor registrado $\left(83^{\circ}\right)$, mostrando que cuando hay una pequeña variación en los valores del componente $3^{\circ} \mathrm{A}$, de $5,6 \%$ a $7,5 \%$ (B1 a B5), no siempre queda caracterizada la disminución del desfase. El pararrayos $\mathrm{B} 6$ se destaca de los demás del fabricante $B$, pues presentó valores elevados de la corriente de fuga total y del componente $3^{\circ} \mathrm{A}(18 \%)$, siendo obtenido el menor desfase $\left(51^{\circ}\right)$. El pararrayos B6 fue aprobado en el ensayo de tensión disruptiva a la frecuencia industrial; sin embargo, presentó resultados insatisfactorios cuando sometido a otras técnicas de diagnóstico, cuyo análisis será presentado más adelante.

\section{Medición de RIV y termovisión}

Los resultados de las mediciones de RIV y termovisión son presentados en la Tabla 4. Para facilidad del análisis, son mostrados también los valores obtenidos de tensión disruptiva a la frecuencia industrial $(60 \mathrm{~Hz})$ y de la medición de la corriente de fuga. Con relación a las mediciones de la corriente de fuga, son relacionados los valores de pico $\left(I_{p}\right)$, el componente $3^{\circ}$ A y el defasaje $(\Psi)$ entre la tensión aplicada $(60 \mathrm{~Hz})$ y la corriente de fuga total $(60 \mathrm{~Hz})$. Los parámetros de la Tabla 4 son: $(R)$ es la indicación de pararrayos reprobados en el ensayo de tensión disruptiva a la frecuencia industrial; (a) indica que el pararrayos presentó radiointerferencia menor que aquél proveniente del ambiente y (b) indica que la medición no fue realizada en el pararrayos. Los valores en la Tabla 4 son presentados, para cada fabricante, en orden creciente del componente $3^{\circ} \mathrm{A}$ de la corriente de fuga, con excepción de los resultados de los pararrayos A5 y A6.

En cuanto a la termovisión, son mostrados los valores Tmáx, que es la mayor temperatura encontrada en el pararrayos sobre la línea LI01, y $\Delta T$ Max que se refiere a la máxima diferencia de temperatura sobre la línea LI01. Los niveles de radiointerferencia de los pararrayos A6, B5 y B6 
presentaron valores elevados, indicando la presencia de descargas eléctricas internas. Esos niveles superan aquellos establecidos en la IEC 60099-1 (1991), la cual especifica el valor máximo de $1000 \mu \mathrm{V}$. Los pararrayos A3, A4, A5, B1 y B2 presentaron niveles bajos. Con relación a la termovisión, puede ser observado que, de entre los pararrayos $A 1, A 2$ y A5 (fabricante $A$ ), el A5 presentó mayor calentamiento. Considerándose los pararrayos B3, B5 y B6 (fabricante B), el B6 presentó calentamiento bastante acentuado.

Tabla 4: Comparación de los resultados.

\begin{tabular}{|c|c|c|c|c|c|c|c|}
\hline \multirow{2}{*}{ Pararrayos } & \multirow{2}{*}{$\begin{array}{c}\text { Tensión } \\
\text { disruptiva a la } \\
\text { frecuencia } \\
\text { industrial }(\mathrm{kV})\end{array}$} & \multicolumn{3}{|c|}{ Corriente de fuga (mA) } & \multirow{2}{*}{$\begin{array}{l}\text { RIV } \\
(\mu \mathrm{V})\end{array}$} & \multicolumn{2}{|c|}{ Termovisión $\left({ }^{\circ} \mathrm{C}\right)$} \\
\hline & & $I_{p}$ & 3으 A (\%) & $\begin{array}{c}\Psi \\
\text { (grados) }\end{array}$ & & Tmáx & $\Delta T$ Máx \\
\hline $\mathrm{A} 1$ & $134(\mathrm{R})$ & 0,172 & 6,7 & 89 & (a) & 20,8 & 2,0 \\
\hline $\mathrm{A} 2$ & $105(\mathrm{R})$ & 0,213 & 10,1 & 65 & (b) & 21,6 & 2,0 \\
\hline A3 & $85(\mathrm{R})$ & 0,456 & 24,9 & 54 & 11 & (b) & (b) \\
\hline A4 & $102(\mathrm{R})$ & 0,791 & 32,9 & 47 & 29 & (b) & (b) \\
\hline A5 & $(\mathrm{R})$ & 0,357 & 5,7 & 39 & 143 & 24,2 & 5,1 \\
\hline A6 & $(\mathrm{R})$ & 0,348 & 5 & 67 & 8033 & (b) & (b) \\
\hline B1 & 226 & 0,396 & 5,6 & 73 & 23 & (b) & (b) \\
\hline B2 & 219 & 0,501 & 5,8 & 75 & 80 & (b) & (b) \\
\hline B3 & 224 & 0,346 & 6,8 & 79 & (a) & 20,8 & 2,9 \\
\hline B4 & 218 & 0,362 & 6,9 & 68 & (a) & (b) & (b) \\
\hline B5 & $188(\mathrm{R})$ & 0,430 & 7,5 & 83 & 4518 & 20,0 & 2,7 \\
\hline B6 & 233 & 0,823 & 18 & 51 & 6381 & 33,7 & 17,6 \\
\hline
\end{tabular}

Las siguientes observaciones pueden ser hechas cuando los resultados son analizados conjuntamente:

Fabricante A:

1) el pararrayos $A 1$ presentó el mayor valor de tensión disruptiva a la frecuencia industrial y el menor valor de la corriente de fuga, de entre los pararrayos de ese fabricante. No presentó niveles de radiointerferencia y calentamiento acentuado en la termovisión;

2) en los pararrayos que presentaron comportamiento estable en la determinación de la tensión disruptiva a la frecuencia industrial (A1, A2, A3 y A4), fue observado que el aumento de la amplitud y del componente $3^{\circ} \mathrm{A}$ de la corriente de fuga, no resulta necesariamente en la disminución, en la misma proporción, de los valores de tensión disruptiva a la frecuencia industrial;

3) el desfase entre tensión aplicada y corriente de fuga total del pararrayos $A 1$ fue de $89^{\circ}$, pudiendo ser notado que todos los otros pararrayos presentaron desfases bastante menores;

4) los pararrayos A5 y A6, que presentaron problemas en la determinación de la tensión disruptiva a la frecuencia industrial, mostraron niveles más elevados de radiointerferencia, en especial el pararrayos A6;

5) de entre los pararrayos sometidos a la termovisión, el A5 presentó calentamiento mayor que A1 y A2. Se observa también que la corriente de fuga del pararrayos A5 fue superior a la de A1 y A2.

Fabricante B:

1) el pararrayos $B 5$, reprobado en el ensayo de tensión disruptiva a la frecuencia industrial, presentó componente $3^{\circ}$ A $(7,5 \%)$ ligeramente mayor que los pararrayos B1 a B4 $(5,6$ a 6,9\%). En B5 fue observado un nivel de radiointerferencia muy elevado $(4.518 \mu \mathrm{V})$. No fue detectado calentamiento 
acentuado y los valores de temperatura, determinados por el termovisor, quedaron muy próximos de aquellos presentados por B3;

2) el pararrayos B6 fue aprobado en el ensayo de tensión disruptiva a la frecuencia industrial, indicando el funcionamiento correcto de los centelladores; sin embargo, presentó valores muy elevados de corriente de fuga y del componente $3^{\circ}$ A. Presentó también un nivel muy elevado de radiointerferencia y calentamiento excesivo;

3) en cuanto a los otros pararrayos (B1 a B4), los valores de la corriente de fuga y del componente $3^{\circ} \mathrm{A}$ quedaron muy próximos. Los pararrayos B1 y B2 presentaron menores niveles de radiointerferencia.

El ensayo de tensión disruptiva a la frecuencia industrial propició resultados importantes respecto del funcionamiento de los centelladores, mientras que el ensayo de tensión disruptiva de impulso atmosférico consideró todos los pararrayos aprobados. Esta última técnica comprueba el comportamiento de los centelladores frente a las ondas impulsivas, no siendo posible evaluar el estado general del pararrayos. La aprobación delo pararrayos en el ensayo de tensión disruptiva a la frecuencia industrial no significa que el mismo esté en buen estado. Las dos técnicas son de difícil ejecución en el campo.

La medición de la corriente de fuga propició informaciones valiosas respecto del estado del pararrayos, especialmente cuando son registrados valores elevados del componente $3^{\circ} \mathrm{A}$, indicando mayor degradación del pararrayos. La medición del desfase entre la tensión aplicada $(60 \mathrm{~Hz})$ y la corriente de fuga total $(60 \mathrm{~Hz})$ puede adicionar informaciones importantes respecto del estado de los pararrayos, mejorando el diagnóstico de estos equipos. El nivel de radiointerferencia indicó pararrayos con descargas eléctricas internas, siendo que algunos pararrayos deteriorados serían identificados apenas por esta técnica. La medición de la radiointerferencia en la subestación requiere cuidados adicionales en función de interferencias que pueden ocurrir en la frecuencia de medición. La termovisión ha sido muy utilizada por las concesionarias brasileñas, pero requiere cuidados en la ejecución y el análisis de los resultados. Esta técnica acusó calentamiento acentuado en algunos pararrayos, aunque no fue capaz de identificar todos aquellos deteriorados.

De modo general, la medición de la corriente de fuga permitió observar que hay una relación entre los valores más elevados de amplitud y del componente $3^{\circ} \mathrm{A}$ y la existencia de degradación en el pararrayos. La presencia de descargas eléctricas en el interior del pararrayos fue verificada a través de la medición de radiointerferencia. La utilización conjunta de la termovisión, radiointerferencia y corriente de fuga total, con el análisis del componente $3^{\circ} \mathrm{A}$, mostró que es posible identificar los pararrayos de $\mathrm{SiC}$ con degradación. Los valores de amplitud de la corriente de fuga y del componente $3^{\circ} \mathrm{A}$ pueden propiciar mayores detalles en cuanto a diferentes estados de degradación, cuando son comparadas mediciones de pararrayos del mismo fabricante y familia.

\section{Medición de la corriente de fuga en la subestación de Paraibuna}

Las mediciones fueron realizadas en pararrayos de $\mathrm{SiC}$, clase de tensión $88 \mathrm{kV}$, y tuvieron por objetivo evaluar la aplicación de esta técnica en el campo. En la subestación, el pararrayos poseía un contador de descarga. En las mediciones fue utilizado un equipo disponible comercialmente, siendo compuesto de un transformador de corriente (TC) y del instrumento de medición, donde era posible efectuar las lecturas de la corriente de fuga total en la frecuencia de $60 \mathrm{~Hz}$ (pico y eficaz), y en la frecuencia de $180 \mathrm{~Hz}$ (valor eficaz), obteniendo así el componente $3^{\circ} \mathrm{A}$. La Figura 1 muestra el esquema utilizado en la medición. Las mediciones fueron realizadas con el TC en la posición 1. Cuando el TC era colocado en la posición 2, había también el registro de las corrientes inducidas entre el cable de tierra y las grampas de la base metálica. Esas corrientes no son provenientes de los pararrayos. La Tabla 5 muestra los valores eficaces de la corriente de fuga, con el TC en la posición 1, obtenidos en las mediciones de los pararrayos de SiC de los circuitos TAU-01, JAG-01 y JAG-02.

Durante las mediciones de la corriente de fuga de los pararrayos, fue también registrada la forma de onda de la corriente de los pararrayos, a través del TC, que posee salida hacia el osciloscopio, y de la tensión del sistema a través de un sensor de campo. Los valores de corriente de fuga de los pararrayos muestran correspondencia con aquellos encontrados en el laboratorio. La realización de 
mediciones periódicas para acompañar la evolución de la corriente de fuga, en términos de amplitud y componente $3^{\circ} \mathrm{A}$, podrá indicar la existencia de algún proceso de degradación más acentuado en los pararrayos de SiC.

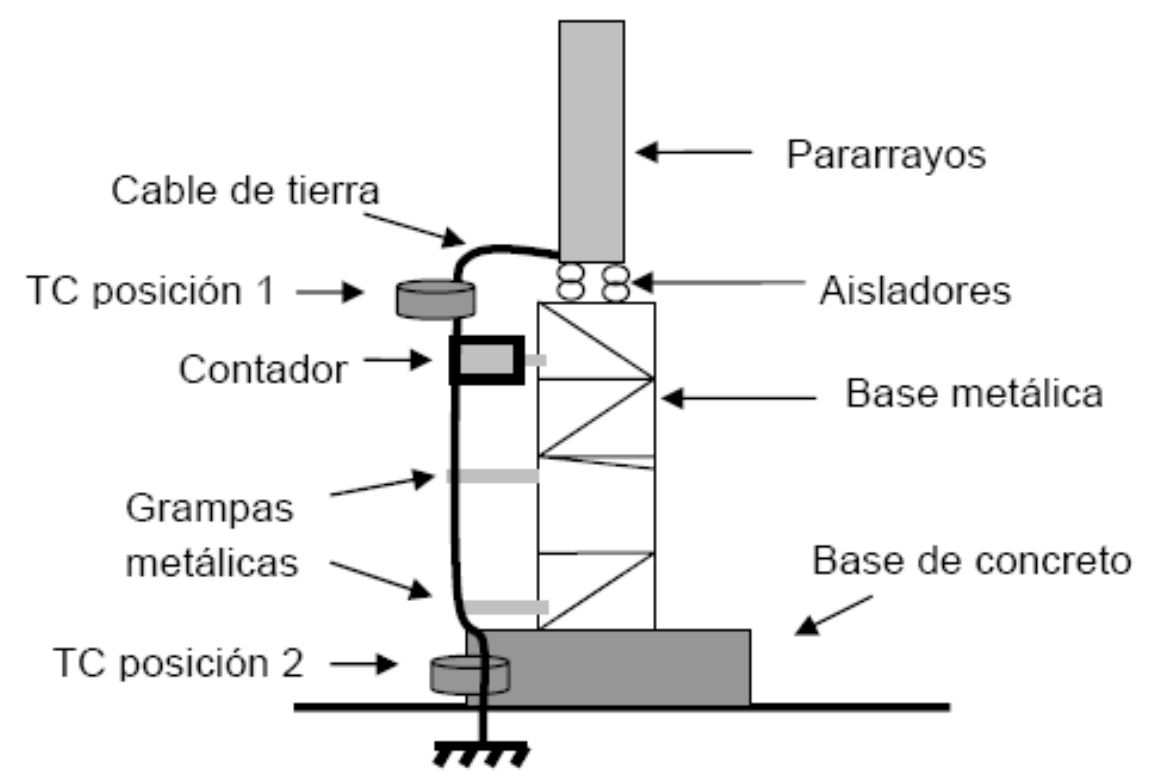

Fig. 1: Esquema de medición de los pararrayos de SiC en la subestación de Paraibuna.

Tabla 5: Valores eficaces de la corriente de fuga (mA).

\begin{tabular}{|c|c|c|c|}
\hline \multirow{2}{*}{ Circuito } & \multicolumn{3}{|c|}{ Corriente de fuga (mA) } \\
\cline { 2 - 4 } & $(60 \mathrm{~Hz})$ & $(180 \mathrm{~Hz})$ & $3^{\circ} \mathrm{A}(\%)$ \\
\hline TAU-01a & 0,132 & 0,005 & 3,8 \\
\hline TAU-01b & 0,128 & 0,004 & 3,1 \\
\hline TAU-01c & 0,112 & 0,004 & 3,6 \\
\hline JAG-01a & 0,170 & 0,004 & 3,6 \\
\hline JAG-01b & 0,078 & 0,002 & 2,4 \\
\hline JAG-01c & 0,163 & 0,005 & 3,1 \\
\hline JAG-02a & 0,122 & 0,003 & 2,5 \\
\hline JAG-02b & 0,088 & 0,002 & 2,3 \\
\hline JAG-02c & 0,070 & 0,002 & 2,9 \\
\hline
\end{tabular}

\section{CONCLUSIONES}

En este trabajo fueron realizados ensayos en el laboratorio en pararrayos de carburo silicio, además de medición en la subestación de la concesionaria. Con base en los resultados obtenidos, las principales conclusiones son:

a) la medición de la corriente de fuga permitió observar que hay una relación entre los valores más elevados de amplitud y del componente $3^{\circ}$ A y la existencia de degradación en el pararrayos; 
b) los valores de la corriente de fuga pueden propiciar mayores detalles en cuanto a diferentes estados de degradación, cuando son comparadas mediciones de pararrayos del mismo fabricante y familia;

c) la medición en la subestación mostró que es posible obtener los valores de la corriente de fuga en pararrayos en servicio.

\section{AGRADECIMENTOS}

Los autores agradecen a la Companhia de Transmissão de Energia Elétrica Paulista (CTEEP) por el soporte financiero a la investigación.

\section{REFERENCIAS}

Almeida, C. A. L. y otros seis autores, Intelligent Thermographic Diagnostic Applied to Surge Arresters: A New Approach, IEEE Transactions on Power Delivery, 24 (2), 751-757 (2009).

Carneiro, J. C., Policy for Renewal of Power System Substations Silicon Carbide (SiC) Surge Arresters: A New Technical Economical Vision, International Symposium on Lightning Protection, 294-299, Foz do Iguaçu, Brazil, $26^{\text {th }}-30^{\text {th }}$ September (2007).

Christodoulou, C. A. y otros tres autores; Simulation of Metal Oxide Surge Arresters Behavior, IEEE Power Electronics Specialists Conference (PESC 2008), 1862-1866, Rhodes, Greece, 15-19 June (2008).

Heinrich, C. y V. Hinrichsen, Diagnostics and Monitoring of Metal-Oxide Surge Arresters in HighVoltage - Comparison of Existing and Newly Developed Procedures, IEEE Transactions on Power Delivery, 16 (1), 138-143 (2001).

IEC 60099-1, Surge arresters - Part 1: Non-Linear Resistor Type Gapped Surge Arresters for A.C. Systems - Edition 3.1 (1999).

IEC 60099-5, Surge arresters - Part 5: Selection and Application Recommendations - Edition 1.1, 3-6 (2000).

Kannus, K. y K. Lahti, Evaluation of the Operational Condition and Reliability of Surge Arresters Used on Medium Voltage Networks, IEEE.Transaction on Power Delivery, 20 (2), 745-750 (2005).

Miyakawa, Y. y otros tres autores; Influence of Temperature Variation on Characteristic of ZnO Elements, Proceedings of 2008 International Symposium on Electrical Insulating Materials, 119-122, Yokkaichi, Japan, 7-11 September (2008).

NBR 5287: Pára-raios de Resistor Não Linear a Carburo de Silicio (SiC) para Circuitos de Potência de Corriente Alternada - Especificação, Rio de Janeiro, Brasil, (1988).

Piantini, A. y J. M. Janiszewski, Efectos de las Ramificaciones y de los Edificios en los Voltajes Inducidos por Rayos en Líneas de Distribución Urbanas, Información Tecnológica, 15 (6), 51-58 (2004).

PSpice Schematic, Version 9.1, http://www.cadence.com. Acceso: 12 de Deciembre (2008).

Schei, A., Diagnostics techniques for surge arresters with main reference to on-line measurement of resistive leakage current of metal-oxide arresters, International Conference on Large High Voltage Electric Systems (CIGRÉ 2000), 1-10, 2000, August 27 - September 1, Paris, France (2000). 